\title{
Why so serious? - Comparing two traffic conflict techniques for assessing encounters in shared space
}

\author{
CARLA JAKOBOWSKY $^{\mathrm{a}, \mathrm{b}}$, FELIX W. SIEBERT ${ }^{\mathrm{b}, \mathrm{c}}$, CAROLINE SCHIESSL $^{\mathrm{d}}$, MAREK JUNGHANS $^{\mathrm{e}}$ MANDY DOTZAUER $^{\mathrm{e}}$
}

\author{
a. iTec, RWTH Aachen University, 52062 Aachen, Germany \\ b. Technische Universität Berlin, 10623 Berlin, Germany \\ c. Friedrich-Schiller-University Jena, 07743 Jena, Germany \\ d. German Aerospace Center, 38108 Braunschweig, Germany \\ e. German Aerospace Center, 12489 Berlin, Germany
}

evasive action, and distance-to-collision (DTC). Trajectory and video data of a shared space were recorded using the Application Platform for Intelligent Mobile Units (AIM) in Ulm, Germany. 1364 interactions were randomly selected. Due to different exclusion criteria, such as interaction partners not being a car or pedestrian, missing values, and detection errors, 69 encounters were available for analyses. Using the PVCA, nine encounters were classified as critical and 60 as non-critical interactions. In contrast, computing the values based on the STCT, only three of the 69 encounters were categorised as critical. The results of a Spearman rank correlation did not show a significant correlation between the severity categories of the PVCA and severity levels of the STCT $(r=0.03, p=0.78)$. An additional analysis of the encounters ranked as critical by the PVCA but as non-critical by the STCT showed that all six encounters had a large temporal distance $(>2 \mathrm{~s})$ combined with very small spatial distance $(<5 \mathrm{~m}$ for vehicles and $<2.5 \mathrm{~m}$ for pedestrians). While the PVCA and STCT yielded similar results in most encounters, this could not be confirmed for all. Results indicate that spatial distance may contribute to the severity of encounters between pedestrians and vehicles in a shared space.

\section{INTRODUCTION}

In 2019, approximately 2.7 million road crashes occurred in Germany, marking an increase of almost $2 \%$ in comparison to the previous year (Statistisches Bundesamt [Destatis], 2020). Vulnerable road users (VRU), meaning road users such as pedestrians and cyclists not protected by a hardened chassis, are especially at risk of being fatally injured as a result of a road crash. Researchers found that car occupants have a markedly lower risk of fatal injury when compared to pedestrians (Follmer \& Gruschwitz, 2019; Destatis, 2018). Since pedestrians' biological crash resistance cannot easily be improved, road safety actors aim to increase pedestrians' safety through road infrastructure design, for example, by separating pedestrian paths from car traffic (World Health Organization., 2009). However, since the traffic infrastructure has historically developed to accommodate the growing number of cars, this separation of pedestrian and car traffic is often difficult to achieve (World Health Organization., 2009).

In contrast to approaches aiming to separate car and pedestrian traffic, Hans Monderman proposed an infrastructure solution to increase the safety of VRUs by not separating, but intentionally integrating the different types of road users in the same space (Monderman, Clarke, \& Baillie, 2006). This street design concept of 'shared space' is characterised by traffic sign reduction and the need for cooperation between road users (Hamilton-Baillie \& Jones, 2005). As a result, all road users in the shared space have to be considerate and pay attention to others, which is a stark reversal of established priorities, creating a road environment in which motorised vehicles are especially required to consider other road users' rights. Consequentially, research shows decreasing velocities in shared spaces, indicating that the concept has some merit (Hamilton-Baillie, 2008; Karndacharuk, Wilson, \& Dunn, 2014a; Monderman et al., 2006). An effect of the number of pedestrians was also revealed. Large numbers of pedestrians related to stronger reductions in the velocity of motorised vehicles (Karndacharuk, Wilson, \& Dunn, 2013). For overall road safety, studies indicate a positive effect on road safety, with reduced numbers of crashes in shared spaces (Hamilton-Baillie \& Jones, 2005). But while this reduction in crashes is a positive indicator for road safety, traffic crashes are rare, and their overall frequency is an insufficient variable to comprehensively assess the safety of the shared space concept, as other interactions not resulting in a collision, need to be assessed as well. For the purpose of analysing the safety related encounters of road users, Kaparias et al. (2010) developed the pedestrian-vehicle conflicts analysis (PVCA), used to assess interactions between pedestrians and vehicles (for further details see Section 2.2). As part of the PVCA's implementation Kaparias et al. (2010) compared the PVCA with the well-established Swedish traffic conflicts technique (STCT) 
from Hydén (1987) and came to the conclusion that these two methods rate road safety-related criticality of interactions between pedestrians and vehicles similarly. However, to date, the relationship between these two methods and resulting differences and similarities have not been comprehensively analysed. Hence, we conducted a study to systematically analyse interactions between pedestrians and vehicles encountering each other in a shared space in Ulm, Germany applying Kaparias' PVCA and Hyden's STCT approach. The goal of this study is to investigate the relationship between the PVCA and STCT, identify similarities and differences, and extract factors describing the criticality of encounters between vehicles and pedestrians in shared spaces.

\section{THEORETICAL BACKGROUND}

\subsection{Shared space}

In the late 1970s, Hans Monderman was assigned the task of implementing measures to reduce traffic on the main street in the Dutch village Oudehaske. Instead of using traditional traffic calming measures, such as speed bumps, Modermann decided to remove all road signs and markings so that the street looked more like a "simple, pretty village" (Monderman et al., 2006, p. 290). As result, average traffic speed was reduced from almost $60 \mathrm{~km} / \mathrm{h}$ to $30 \mathrm{~km} / \mathrm{h}$ : A 40 per cent decrease in speed (Engwicht, 2005; Hamilton-Baillie, 2008). After building other shared spaces in other villages and towns, similar positive effects on speed reduction were observed (Hamilton-Baillie, 2008; Hamilton-Baillie \& Jones, 2005; Karndacharuk et al., 2014a). According to Monderman, the effect is related to how drivers perceive the street design. As they drive into a street that looks more like a small village road and less like a main street, car drivers adjust their behaviour accordingly. Drivers start to interact with the pedestrians and bicyclists and thus become "an integral part of the social and cultural context" of the road environment (Monderman et al., 2006, p. 291).

Even though the shared space street design concept is several decades old, well-established and frequently used, distinct determinative definition is not established. Instead, many different definitions can be found in the literature (Karndacharuk et al., 2014a; Moody \& Melia, 2011; Schönauer, Stubenschrott, Schrom-Feiertag, \& Menšik, 2012). Gerlach, Boenke, Leven, and Methorst (2008) posit that shared spaces always need to be integrated into the existing traffic situation resulting in tailor-made solutions. Even though shared spaces are not uniformly designed, a common understanding of the design principles of shared spaces exists. According to these common principles, traffic signs, traffic lights, or road markings need to be reduced to a minimum (Gerlach et al., 2008; Hamilton-Baillie \& Jones, 2005). Also, speed limits should be reduced to $30 \mathrm{~km} / \mathrm{h}$ or lower to foster eye contact between road users (Hamilton-Baillie \& Jones, 2005). Generally, the main idea of shared spaces is to create a traffic environment that resembles a social area rather than a street situation (Engwicht, 2005). This appearance may explain the positive effects on velocity. In addition, Karndacharuk et al. (2013) found that the number of pedestrians correlated with the speed choice of car drivers. As the number of pedestrians increased, speed decreased fitting to the phenomenon of 'safety in numbers' (Jacobsen, 2015). Furthermore, research shows that not only the number of crashes decreased compared to the traffic situation before remodelling it to a shared space, but the number of conflicts between pedestrians and vehicles as well (Hamilton-Baillie \& Jones, 2005; Kaparias et al., 2013; Monderman et al., 2006).

\subsection{Traffic conflict techniques}

Shared spaces are designed to reduce traffic hazards and increase safe interactions between different road users. To systematically analyse interactions between pedestrians and vehicles, standardised methods are needed determining the severity of interactions between road users. In 1987, Hydén developed such a method: The Swedish traffic conflicts technique (STCT) used to grade interactions between motorised vehicles in general road traffic situations. The STCT consists of two factors: Time-to-accident (TA) and conflicting speed (CS). TA is the remaining time of the evading vehicle until the potential collision point. CS is the speed of the evading vehicle at the start of the evading action. After the calculation of the two factor values, a diagram is used to determine the severity of the encounter (see Figure 1). In total, 30 severity levels are defined, all levels higher than or equal to 26 are considered critical. When both interaction partners evade (dual-evading encounters), the lower of both scores is used to classify the situation (Laureshyn \& Varhelyi, 2018).

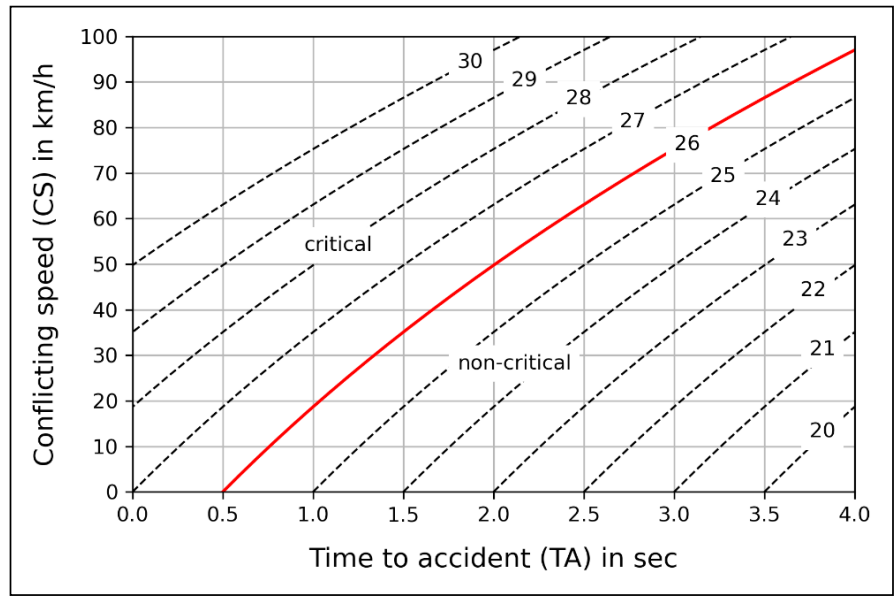

Figure 1. Conflict severity diagram for the STCT (after Laureshyn \& Varhelyi, 2018, p. 5).

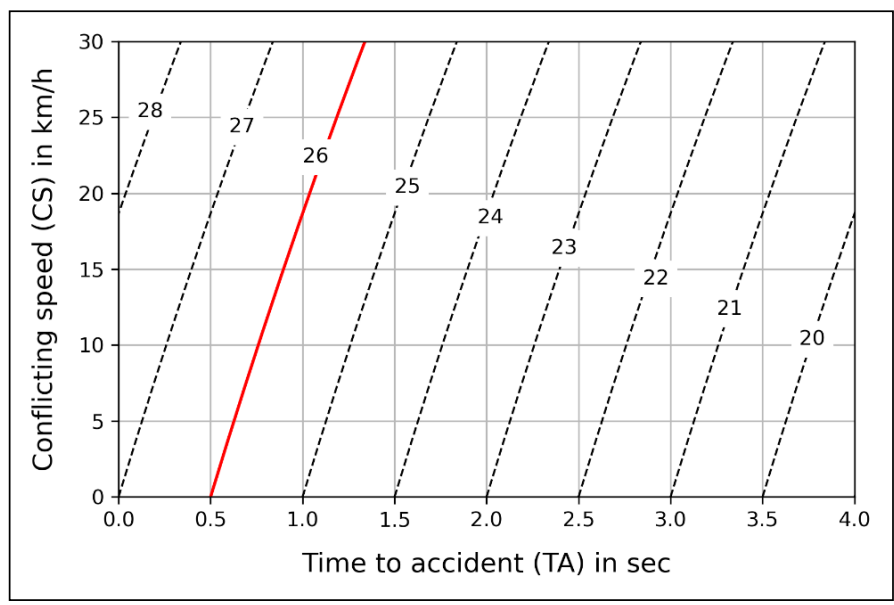

Figure 2. Extract from the conflict severity diagram up to a maximum of $30 \mathrm{~km} / \mathrm{h}$.

As the main speed range in shared space is below $30 \mathrm{~km} / \mathrm{h}$, Figure 2 shows the corresponding section of the conflict severity diagram for speeds up to a maximum of $30 \mathrm{~km} / \mathrm{h}$. A first glimpse reveals that the impact of CS in this speed range is distinctly lower than that of TA. In particular, below $20 \mathrm{~km} / \mathrm{h}$ (speed mainly adapted by pedestrians), the TA value is decisive for the degree of severity. According to STCT, all situations with TA values smaller than $0.5 \mathrm{~s}$ are considered as critical when speed is below $30 \mathrm{~km} / \mathrm{h}$. All situations with a TA greater than $1.3 \mathrm{~s}$ are automatically categorised as noncritical regardless of CS. Only in the TA range of $0.5-1.3 \mathrm{~s}$, CS determines the categorisation of situations.

In contrast to the STCT, the pedestrian vehicle conflicts analysis (PVCA), developed by (Kaparias et al., 2010) takes 
the distinct characteristics of road users into account and is therefore potentially better suited to analyse encounters in shared space environments. It is based on the institute of highways and transportation conflicts technique (IHTCT, Swain, 1987) and was revised three years later (Kaparias et al., 2013). The method consists of four factors: (A) time-tocollision (TTC), (B) severity of evasive action, (C) complexity of evasive action and (D) distance-to-collision (DTC). Every factor contains between two and four levels. For some factors, the definition of the levels differs depending on type of road users in order to consider the specifics of different road user types (see Table 1).

In the PVCA, a table system is used to categorise the severity of the encounter ranging from (1) slight to (4) critical after determining all factors for each pedestrian-vehicle encounter (see Appendix A). In dual-evading encounters, individual severity ranks for each evading road user are calculated and the higher score is used for the categorisation (Kaparias et al. 2010). A closer look at the table system (Appendix A) reveals that the four factors influence the severity classification differently. As an example, Factor C (complexity of evasive action) has only little to almost no influence on the classification of the interactions. Only when TTC is moderate (level 2 in Factor A), severity of evasive action slight (level 1 in Factor B), and DTC moderate (level 2 in Factor D), the complexity of the evasive action determines the degree of severity. In all other factor combinations, Factor $\mathrm{C}$ does not lead to any changes in ratings from critical to slight or vice versa.

Kaparias et al. validated the PVCA method on an urban road in London before and after its transformation to a shared space using severity ratings of the STCT as a comparison (Kaparias et al., 2010; 2013). In their analysis, they concluded "that the two methods perform similarly well" (Kaparias et al., 2010, p. 81), and slight differences between ratings are prescribed to different focuses on road users. Therefore, one could expect a high relationship between the rankings of the two methods. However, a more fundamental comparison of the variables used in both methods reveals not only similarities but also differences that might influence the ranking. In both methods, the moment of the evasive action is used as a temporal anchor for the calculation of the severity of the interaction. Additionally, both methods categorise encounters as either critical or non-critical/slight. With regard to difference, the main divergence relates to the number of factors that are used to arrive at the severity rating. The PVCA consists of four factors, related to the temporal (TTC) as well as spatial distance (DTC) between road users, and the severity and complexity of the evasive action. In the STCT, only the temporal distance and the speed of the evading interaction partner are considered. Another difference between the methods is the inclusion of specific variable levels for different types of road users. While the PVCA was developed for encounters between pedestrians and vehicles in shared spaces, the main focus of the STCT is on encounters between pairs of vehicles in more traditional road environments. As a last difference, the procedure to integrate the severity of encounters in which both road users take an evasive action is handled differently. While the PVCA utilises the rating of the road user that is more critical, the STCT uses the less critical rating to arrive at the severity rating of an encounter. In conclusion, at this point, it is not clear whether the differences in classification result from the different focus as Kaparias et al. (2010) hypothesised or because of the selection of the factors.

\subsection{Research questions and aim of this study}

In light of the apparent differences between the PVCA and the STCT, as well as a lack of a detailed comprehensive analysis of their variables, the aim of the current study is to compare the classifications of interactions between pedestrians and car drivers in a shared space as they are rated for their severity by the PVCA and STCT. In addition, factors describing the individual rankings (i.e. factors that potentially relate to the severity of encounters) will be identified. To investigate our research questions, video and trajectory data collected in a shared space in the city of Ulm, Germany is used. Based on the theoretical comparison of the two methods, we generally expect differences in the rankings by the two methods unlike Kaparias et al. (2010), and, considering the different variable sets that are utilised by the two methods, we anticipate these differences for interactions with large temporal distances and small spatial distances. Furthermore, it is expected that interactions with two evading interaction partners are ranked differently by the two methods, due to differences in the integration procedures of the two methods.

\section{METHODS}

\subsection{Data collection}

Between December $12^{\text {th }}$ and $20^{\text {th }} 2016$, video and trajectory data were recorded by the AIM Mobile Unit in a shared space in Ulm, Germany. In this study, only data recorded between December $13^{\text {th }}$ and $19^{\text {th }}$ was used, corresponding to a full week. As the data were recorded in winter time, the data selection was limited to daylight conditions (8 am until 4 pm).

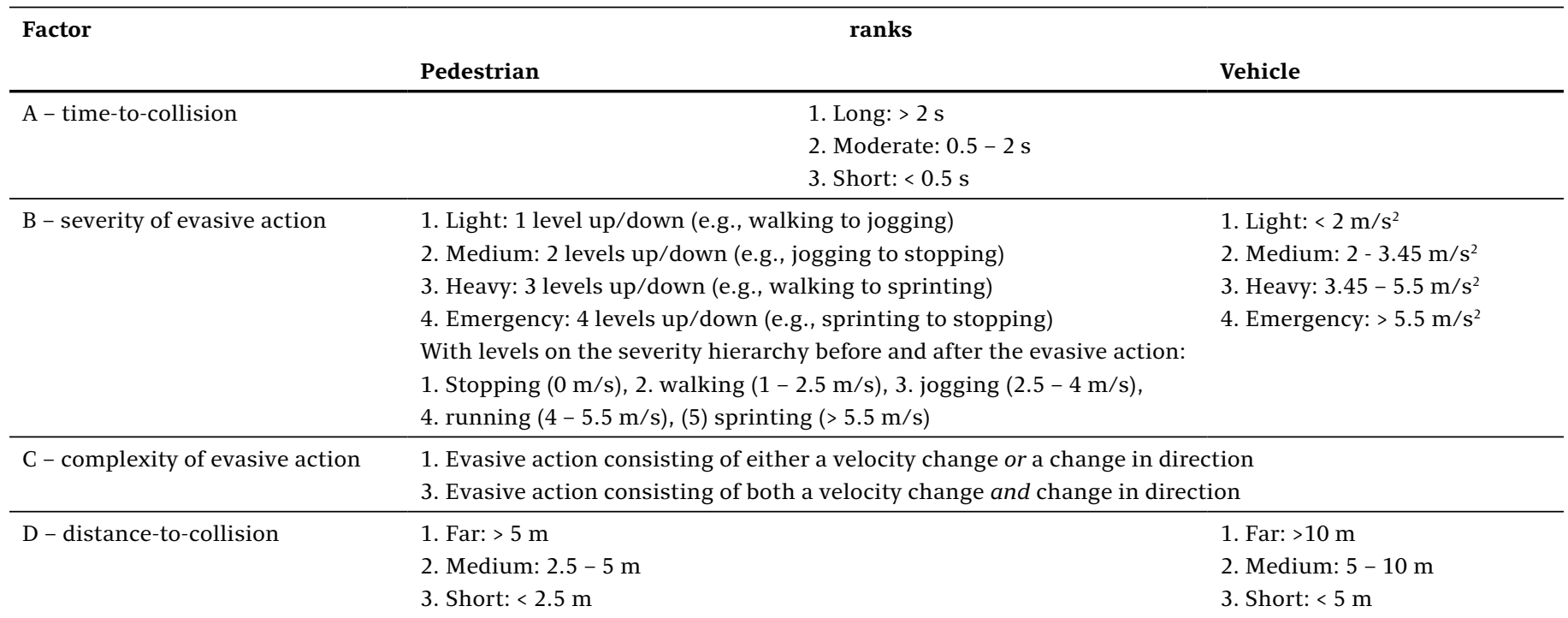

Table 1. Four factors of the PVCA and specifications of ranks (after Kaparias et al., 2013). 
During this period, 3189 potential encounters between car drivers and pedestrians were automatically extracted. 1364 interactions were selected randomly and visually inspected by the authors. According to predefined exclusion criteria (e.g. missing evasive action or interaction partner is not a car driver or pedestrian) situations were either discarded or included in the analysis, resulting in a preliminary dataset of 97 interactions. For these 97 interactions trajectory data were calculated. Due to incorrect detections and missing data, 28 encounters had to be excluded at this stage, resulting in 69 encounters for the final dataset used for analysis.

\subsection{Material \& Apparatus}

AIM Mobile Unit - The video and trajectory data were recorded by the AIM Mobile Unit, a mobile sensory system consisting of a mast, stereo cameras and an infrared flash as well as a weather-proof case containing the different processing computers and other electronical devices. Sensor data were fused and processed in order to produce the main output: detections of road users and trajectories of all detected traffic participants within the view of the AIM unit's sensors. Trajectories contained information about the classification and dimensions of the object, its location, velocity, and other dynamic state variables. They were automatically stored in a database for offline analysis, together with the respective scene videos for manual assessment and validation. Motorised (e.g. car drivers) and non-motorised road users (e.g. pedestrians) were detected, tracked, and classified. For personal data protection the video resolution was reduced by about 95\%. Example frames of video data are presented in Figure 3. Detections and numerical trajectory data were used for analysis.

ELAN Annotation Software - The open source software "EUDICO Linguistic Annotator" (ELAN) version 5.5, a software program for annotating video and audio recordings developed by the Max Planck Institute for Psycholinguistics (Tacchetti, 2018), was used for annotating additional information in the videos. An observation protocol was developed and implemented in order to systematically analyse each interaction. It consisted of demographic information about pedestrians, such as age in five categories (child, adolescent, adult, older person, ambiguous) and gender (male, female, ambiguous), as well as the annotation of the PVCA Factor C - complexity of evasive action (see Section 3.3).

\subsection{Design}

The recorded shared space was located in the Neue Straße at the corner of Marktplatz in Ulm, Germany. It was approx. $250 \mathrm{~m}$ long and consisted of two car lanes and a centre island in between. Because of the placement of the AIM Mobile Unit and the resulting video angle (Figure 3), only interactions between a pedestrian and a motorised vehicle driver were selected that took place in the front area of the left-hand car lane. An interaction was defined as the encounter of a car driver and a pedestrian with a maximum time interval of $2.5 \mathrm{~s}$ (i.e. the trajectories of the road users had intersected within $2.5 \mathrm{~s}$ ).

For the purpose of this study, only situations with an observable evasive action of at least one of the interaction partners were included in the analysis. An evasive action was defined as a change of direction or a change in velocity. For car drivers, a change in velocity was registered as an evasive action when an acceleration or braking was observable, and the average car velocity changed by at least $0.4 \mathrm{~m} / \mathrm{s}^{2}$ (increase or decrease) over a period of $0.7 \mathrm{~s}$ in the period from maximum $3 \mathrm{~s}$ before reaching the point of intersection of the trajectories up until the time of intersection. The duration of $0.7 \mathrm{~s}$ equates the vehicles' braking distance at a speed of $20 \mathrm{~km} / \mathrm{h}$. According to Barbosa, Tight, and May (2000) motorised vehicles show an average velocity change of $\pm 0.4 \mathrm{~m} / \mathrm{s}^{2}$ in a traffic calming area to evade barriers such as speed bumps. The pedestrian's change in velocity was defined similarly: an observable velocity change $>0.4 \mathrm{~m} / \mathrm{s}^{2}$ or $<-0.4 \mathrm{~m} / \mathrm{s}^{2}$ with maximal $3 \mathrm{~s}$ before reaching the trajectory point with the car. The end point of the velocity change was defined as the highest (lowest) value of velocity during the evasive action. The vehicle's change in direction was defined as an observable curve in the trajectory away from the encountering partner (similar to Kaparias et al., 2013). The end of the change in direction was reached when the vehicle was driving straight on again. Similarly, the pedestrian's change in direction was defined as an observable right or left curve in the pedestrian's trajectory. Here, the curve had to turn away from the car lane to be counted as an evasive action. The end of the pedestrian's change in direction was reached when pedestrians walked straight on again.

The PVCA and STCT procedure had to be adapted to fit the purpose of the study. For example, instead of using the point of collision for calculating TTC and DTC, the intersection point was used. All factors that needed to be adapted are listed in Table 2. All other factors were used unchanged. In PVCA and STCT all interactions were ranked into two categories: critical and non-critical for STCT; critical and slight for PVCA. Here, we use the term 'non-critical' for the slight category.

\subsection{Procedure}

The procedure of registering variables of encounters according to the PVCA and the STCT consisted of two parts: (1) video observation and (2) utilisation of trajectory data. All 97 en-
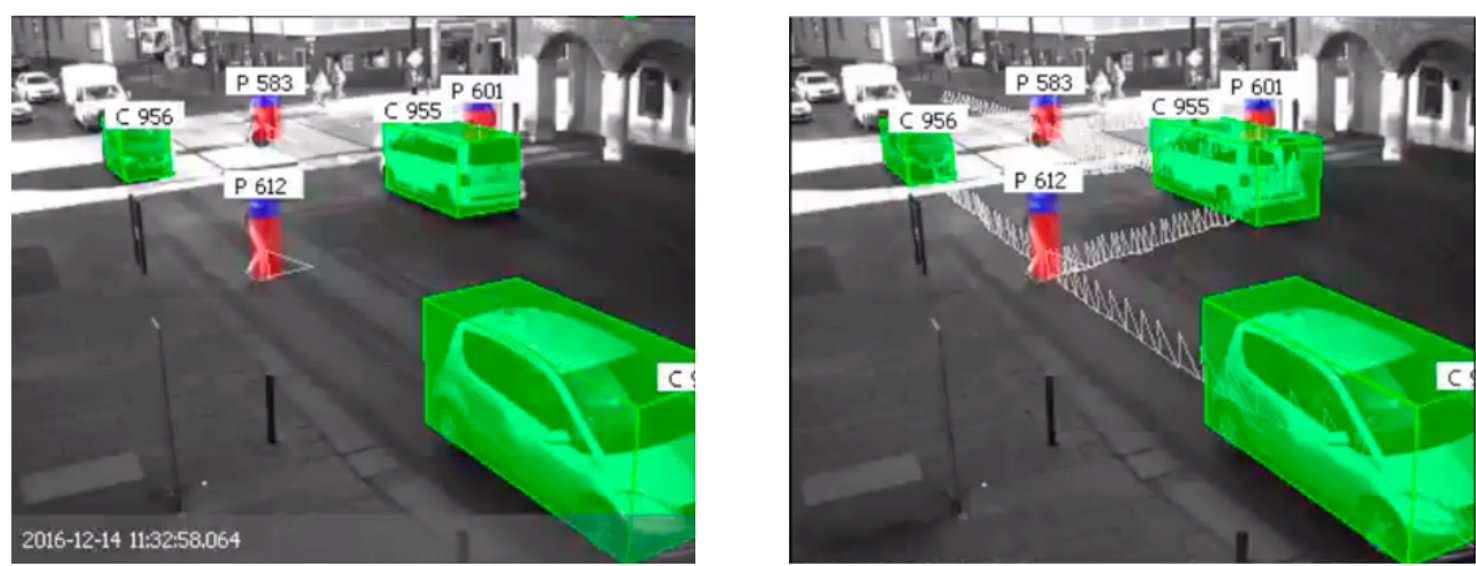

Figure 3. Still frames from video data collected through the AIM unit (left: video frame including classifications of road users; right: video with classifications and trajectories of road users. 


\begin{tabular}{ll}
\hline Original factor & Factor in our study \\
\hline Time-to-accident (TA) & $\begin{array}{l}\text { Time-to-intersection (TTI): Estimated time from the start of the evasive action to the intersection of the } \\
\text { trajectories of both interaction partners under the assumption that they do not change their speed }\end{array}$ \\
\hline Time-to-collision (TTC) & Time-to-intersection (TTI) \\
\hline Distance-to-collision (DTC) & $\begin{array}{l}\text { Distance-to-intersection (DTI): Estimated distance from the start of the evasive action to the intersection } \\
\text { of the trajectories of both interaction partners under the assumption that they do not change their speed } \\
\text { and direction }\end{array}$ \\
\hline Reverity of evasive action & $\begin{array}{l}3 \text { levels up/down, (4) emergency: } 4 \text { levels up/down, with levels on the severity hierarchy before and after } \\
\text { the evasive action: }(1) \text { Stopping }(<1 \mathrm{~m} / \mathrm{s}),(2) \text { walking }(1-2.5 \mathrm{~m} / \mathrm{s}),(3) \text { jogging }(2.5-4 \mathrm{~m} / \mathrm{s}),(4) \text { running } \\
(4-5.5 \mathrm{~m} / \mathrm{s}),(5) \text { sprinting }(>5.5 \mathrm{~m} / \mathrm{s})\end{array}$ \\
\hline
\end{tabular}

Table 2. All factors adapted for this study.

counters of the preliminary dataset were annotated according to the observation protocol. The demographic data of the pedestrians were noted. Additionally, the evasive actions of the interaction partners were assigned to either velocity change, change of direction, or a combination of both by video observation to determine the complexity of evasive action (Factor $\mathrm{C}$ ) of each interaction. If the evasive action was a change in direction, the starting and end point of the action were identified. If the evasive action was a velocity change, the trajectory data of the interactions were processed to identify the starting and end point of the velocity change. Furthermore, the values of TTI, DTI, severity of evasive action and conflicting speed were derived from the trajectory data. After ranking each encounter, the criticality of the encounters was calculated by PVCA and STCT using the table system and conflict severity diagram respectively. In dual-evading encounters, the criticality was calculated for both interaction partners. Afterwards, the most critical PVCA ranking and the least critical STCT ranking were used to determine the interactions' criticality, and the corresponding road user was marked as the evading road user.

For the analysis, the severity rankings of the interactions determined through the PVCA and the STCT were correlated. As the ranks were ordinal scaled and had ties an adaption of the Spearman rank correlation was used, as recommended by Bortz and Schuster (2010). Additionally, differently ranked interactions were further analysed in order to identify potential causes for the differences in rankings, and interactions with two evading road users were investigated. Interrater reliability was calculated for 18 randomly selected encounters (approx. 25\% of encounters).

\section{RESULTS}

In total, 69 interactions were analysed. Of all pedestrians, $36(52 \%)$ were observed as male and 33 pedestrians (48\%) as female. For age categories, 48 pedestrians (70\%) were classified as adults, 12 pedestrians (17\%) as older persons and 9 pedestrians (13\%) as adolescents. No children were observed. In 31 encounters (45\%), the vehicles performed an evasive action. Of these, 24 car drivers decelerated and four changed their direction. Three car drivers decelerated and changed direction in combination. In 27 encounters (39\%), pedestrians performed an evasive action, with 24 pedestrians decelerating and three accelerating. In 11 encounters (16\%), both interaction partners took an evasive action. Here, both interaction partners decelerated in eight situations. In two interactions the pedestrians decelerated and the car drivers changed the direction, whereas in one situation the pedestrian accelerated and the car driver changed the direction. The pedestrians had an average speed of $1.28 \mathrm{~m} / \mathrm{s}(S D=0.56)$ at the moment of the evasive action. The car drivers' mean velocity was $4.01 \mathrm{~m} / \mathrm{s}(S D=1.49)$.
With STCT, 66 encounters were classified as non-critical, meaning their STCT specific level was lower than 26, and three encounters were classified as critical (level equal to or greater than 26). Within the 30 severity levels of the STCT, encounters ranged between 16 and $26(M d n=21)$. In Figure 4, the ranking of the encounters in the conflict severity diagram is visualised, with each dot representing an encounter. Dark blue dots represent situations in which the pedestrian evaded, while light blue dots show dual-evading encounters with the pedestrian as the determined evading partner. Similarly, green dots depict situations in which the car driver did an evasive action, whereas yellow dots display dual-evading encounters in which the car driver was identified as the evader. The dashed lines in the diagram indicate the different levels of severity, with the red line marking the threshold between non-critical (on the right) and critical (on the left). In all critical encounters a pedestrian evaded. The distribution of the evading road users in the 66 non-critical interactions was relatively balanced: 31 pedestrians and 35 car drivers. Although the CS value (Figure 4) only distinguishes between critical and non-critical encounters in the narrow range of TTI between 0.5 and $1.3 \mathrm{~s}$, ten encounters were found within this range, and CS needed to be used to classify encounters. In all these encounters, pedestrians were the evading road users and categorised as non-critical (see Figure 4) due to a low CS.

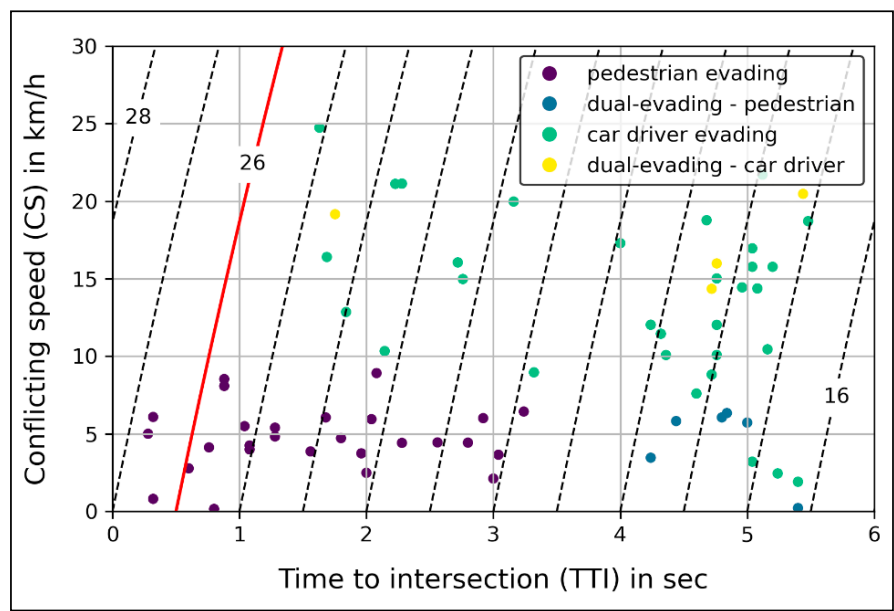

Figure 4. Conflict severity diagram (STCT) filled in with the encounters arranged for conflicting speed (CS) and time to intersection (TTI).

With the PVCA, 60 out of 69 encounters were ranked as non-critical (rank 1). Nine interactions were ranked as critical (ranks 2-3). The ranks were between 1 and $3(M d n=1)$. Out of nine critical ranked encounters, seven interactions were ranked into rank 2 . In all of these seven interactions, the pedestrian evaded. Two encounters were categorised as rank 3. Here, a pedestrian and a car driver evaded. Out of 60 
non-critical encounters, in 25 interactions, the pedestrian was the determined evader, whereas in 35 interactions the car driver was identified as the evading partner. In Figure 5, the relative frequency of the interactions is depicted categorised by the ranks of PVCA and split by the interaction type (dualevading encounter or encounter with one evading partner) and the determined evading partner.

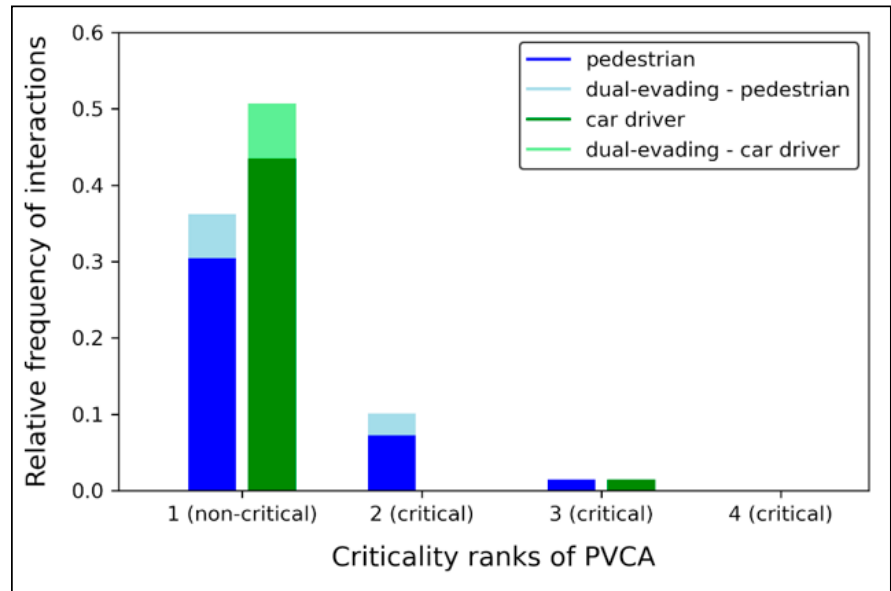

Figure 5. Relative frequency of the interactions ranked by PVCA.

A spearman rank-correlation between the ranks of PVCA and the levels of STCT showed a non-significant correlation $\left(r_{s}=0.03 ; t(67)=0.28 ; p=0.78\right)$. The distribution of the pedestrian-vehicle encounters ranked by the methods is shown in Figure 6. Here, the PVCA data points are plotted on the $\mathrm{x}$-axis and the STCT data points on the $\mathrm{y}$-axis. The thickness of the points is proportional to the number of encounters positioned at the matching data point (see also caption in the upper right corner of Figure 6). At each data point between one and 13 interactions are located. The two red lines divide the encounters into non-critical (left or bottom) and critical (right or top). As one can see, in Figure 6, most interactions are in the non-critical area of both traffic conflict techniques. Three interactions were ranked as critical by PVCA and STCT. All of these interactions had a PVCA-ranking of 2 and a STCTlevel of 26. Six encounters were ranked differently by PVCA and STCT.

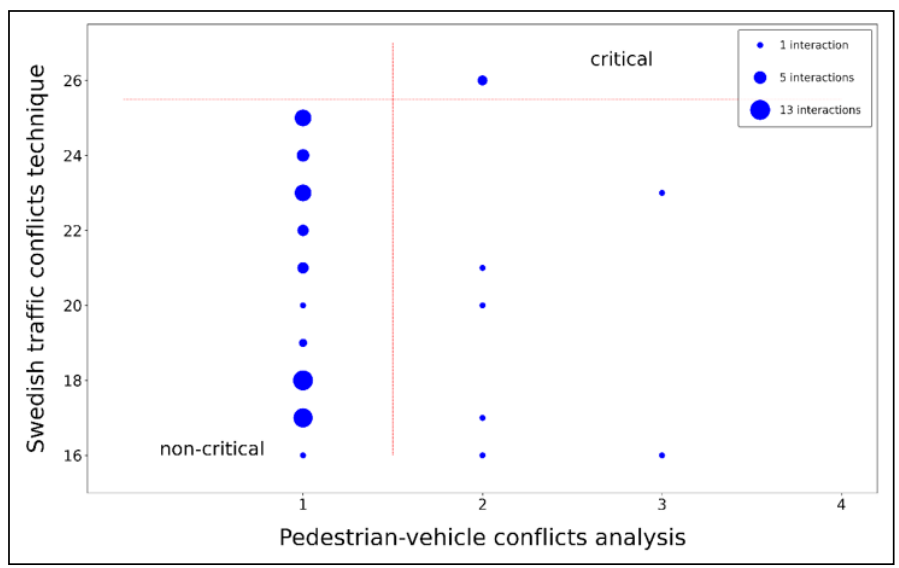

Figure 6. Distribution of the pedestrian-vehicle encounters ranked by PVCA and STCT. Thickness of data points is proportional to the number of situations at the same data point (see caption in the upper right corner).

An additional analysis of the six encounters ranked differently by the two methods, revealed a pattern of relatively high TTI values in combination with small DTI values. As for five encounters, the TTI was higher than $3 \mathrm{~s}$ and for another encounter the TTI was higher than $2 \mathrm{~s}$. At the same time, all encounters were observed to have small DTI values $(<2.5 \mathrm{~m}$ for pedestrians and $<5 \mathrm{~m}$ for vehicles). A further observation of the dual-evading encounters showed that two out of 11 interactions were ranked differently by the methods. Both interactions were ranked as critical through PVCA and as non-critical by STCT. In one of these situations, the different ranking can be attributed to the different handling of the dual-evading encounters. Here, the pedestrian was used for the PVCA ranking and the vehicle was utilised for calculating the STCT ranking. In the other situation, both severity rankings (PVCA and STCT) were done with the pedestrian as the evading road user. Here, through the PVCA the pedestrian's evasive action (rank 2, critical) had a higher rank than the vehicle's evasive action (rank 1, non-critical), whereas through the STCT, the classifications were the opposite (level 16, non-critical, for pedestrian and level 19, non-critical, for motorise vehicle). Through the different handling of the dual-evading encounters, the pedestrian ended up being the evading road user for both interactions. Again, with a high TTI ( $>3 \mathrm{~s})$ and a small DTI $(<2.5 \mathrm{~m})$. Therefore, in this case, the different severity ranking cannot be attributed to the different handling of the dual-evading encounters. All other nine dual-evading encounters were ranked by both methods similarly critical.

To calculate the interrater reliability, the percentage agreement was used. The percentage agreement of the ranking in Factor C of PVCA was $72.7 \%$ for the pedestrians and $87.5 \%$ for the vehicles. The average difference between the identified starting point of the change of direction as evasive action for cars was $0.85 \mathrm{~s}(S D=0.76)$. In one situation one observer found a car changing direction as evasive action which the other did not find. According to one of the observers, three pedestrians changed their direction as evasive action with which the other did not agree. The percentage agreement was $94.4 \%$ for gender and $33.3 \%$ for age of the pedestrians. Data access is possible through the Open Science Framework storage (Jakobowsky, Siebert, Schießl, Junghans, \& Dotzauer, 2021).

\section{DISCUSSION}

The goal of this study was to investigate the relationship between the two traffic conflict techniques STCT and PVCA and to discover factors describing the severity of an encounter between vehicle drivers and pedestrians. Results show that the majority of encounters were ranked similarly by the methods. However, in interactions with a large TTI and a small DTI, interactions were categorised differently. Additionally, only one interaction, in which both road users evaded, was ranked differently, despite different procedures for dual-evading encounters.

\subsection{Comparison of the conflict techniques}

In contrast to Kaparias et al. (2010), we did not find that the two methods performed similarly well. Six out of sixty-nine interactions were ranked differently. In all of these interactions a high TTI (i.e. a relatively long time before the two road users would intersect) was combined with a small DTI (i.e. a small physical distance to the trajectory intersection point). This confirms our expectations stemming from the prior analysis of the variables integrated in the STCT and the PVCA. In addition, we expected that the integration of different road users in case of dual-evading encounters would result in differences in severity ratings. The results indicate that this expectation is not correct, as only one ranking resulted from the utilisation of different evading partners (pedestrian for PVCA and car driver for STCT).

The pattern of high TTI and small DTI in the differently ranked situations may indicate that especially interactions, 
in which road users evaded early but very close to the intersection point, were classified differently. Pedestrians can change speed or direction faster and more abruptly than, for example, car drivers. Therefore, small distances may be a safety risk, especially in shared space, where the traffic behaviour is less predetermined by marked road lanes, curbs, or traffic signs. In low speeds, the ranking by STCT is mainly determined by TTI (see Figure 4), whereas more aspects of the evasive action are considered by PVCA because of four integrated factors. In particular, the factor DTI may be an important gain to get a more comprehensive picture of the criticality of the interaction. Therefore, in low speed and less organized street concepts such as shared spaces, it is not sufficient to only consider TTI to determine the severity level of a vehicle-pedestrian interaction. Factors, such as DTI, appear to be promising for safety traffic analyses. However, as these results are only descriptive, further research is needed to statistically confirm the here found results.

\subsection{Traffic conflict techniques}

PVCA and STCT are traffic conflict techniques used to investigate the criticality of encounters between different road users. With these methods, interactions can be categorised as critical and non-critical. However, both methods are very narrow in their regard to encounters in the road environment (e.g., only encounters with intercepting trajectories are considered), while prospective behaviour that prevents trajectory crossings are not considered. Especially in a road traffic concept such as shared space with road users being less bound by clear traffic rules and thus being able to move more freely, it is inconclusive to what extent a single moment of evading adequately covers the traffic behaviour. In fact, research has already shown that specific conditions such as the number of pedestrians in shared space or previously performed interactions influence the behaviour of pedestrians and car drivers (Karndacharuk et al., 2013, 2014b). Furthermore, Jakobowsky (2020) found preliminary results in line with the phenomenon of 'safety in numbers' (Jacobsen, 2015) as mentioned above (see Section 2.1). The results indicated that car drivers appeared to be more inclined to give right of way to pedestrians when the number of pedestrians present in the shared space was high. However, as these results are only descriptive future research is needed. Thus, a longer inspection of both interaction partners may provide better insight into the dangerousness of situations. Additionally, only the traffic behaviour of the evasive road user is considered in the two analysed methods, although an interaction in the road environment always involves at least two road users. Thus, alternatives that consider not only a single moment but rather the traffic behaviour of both road users over a longer period of time may lead to a better understanding of the interaction's criticality. For instance, Kaparias, Bell, Biagioli, Bellezza, and Mount (2015) developed several qualitative criteria, such as "change in pace", "change in direction" and "subsequent acceleration", to analyse the dynamics of interactions in shared spaces (Kaparias et al., 2015, p. 118). Future research could proceed here to focus on quantified criteria considering elements, such as speed trajectories or braking and acceleration. In this way, the entire dynamics of the shared space can be analysed.

Through the STCT, the severity of each encounter is determined by the temporal distance (TTI) and the speed (CS). However, as shown in Figure 4, the CS factor has a smaller impact on the classification of the encounters than the TTI at velocities below $30 \mathrm{~km} / \mathrm{h}$. Here, only ten interactions were classified as non-critical based on CS (see Figure 4). As already mentioned above, all of the evading road users were pedestrians. Since pedestrians are generally slower than car drivers, encounters with an evading pedestrian may be classified as less critical than encounters with an evading car driver. It may lead to the conclusion that the criticality of interactions with evading pedestrians may be underrated due to the original goal of the STCT analysing interactions between two motorised vehicles. According to Shbeeb (2000) the criticality rating of pedestrian-vehicle encounters becomes more realistic when the focus of the behaviour analyses lies on the car driver. However, in this case, the traffic behaviour of the pedestrian is not considered at all which may lead to loss of information. Especially, if one considers that pedestrians are able to change speed or direction faster and more abruptly than vehicles, and can move more freely in shared space than in other traffic situations. As mentioned above, an approach with a wider focus on both interaction partners may lead to a more comprehensive understanding on the traffic safety in shared spaces. Therefore, it is possible that the TTI and CS alone do not sufficiently predict the severity of pedestrianvehicle interactions in shared space.

Theoretically, the PVCA has advantages over the STCT as it combines four factors considering the temporal and spatial distance as well as the degree and complexity of evasive actions. But similar to the STCT, the PVCA does only consider changes in direction as part of complexity of the evasive action (Factor C). In Factor C, an evasive action is classified as simple, when only a change in direction or a change in velocity was observed, and as complex, when a change in direction and velocity are registered. Correspondingly, the only impact of the change in direction is via Factor $C$ in combination with a velocity change. This might be a relic of the method on which the PVCA was based, as it was developed from the IHTCT (Swain, 1987), as mentioned in Section 2.2, originally developed to analyse conflicts between two vehicles on highways. On highways, the discrimination between these two kinds of evasive actions captures the dangerousness of a car driver changing direction during the evasive action and thereby might losing control over his or her car. However, in shared space, velocity range is below $30 \mathrm{~km} / \mathrm{h}$ and therefore a combination of a velocity change and change in direction might be less critical. Additionally, Factor $\mathrm{C}$ has hardly any influence on the classification of the severity ranks, as it only distinguishes between critical and non-critical encounters for a small number of factor combinations. Therefore, the current situation of Factor C should be reconstructed.

Furthermore, it is conspicuous that the velocity change is part of two factors (Factors $\mathrm{B}$ and $\mathrm{C}$ ), while change in direction is only considered in one factor (Factor C). Thus, the change in speed becomes a more weighting factor than the change in direction. This might be again a relic of the original method IHTCT (Swain, 1987). On highways, velocity changes as evasive actions are more likely to occur than changes in direction due to the clear specification of lanes. In shared space, however, road users can move more freely in all directions. Thus, changes of direction as evasive actions are more likely to appear and should be given more consideration. Therefore, it should be considered to adapt the method with respect to the importance of directional changes. Similar to the degree of velocity change, an inspection of the degree of the change of direction may deliver important information on the severity of the interaction. In general, the intensity and complexity of evasive action should be investigated further, to potentially relate them to subjective factors in road users, such as being scared or surprised. For this, observational data could be combined with brief interviews to further understand the traffic behaviour of the interaction partners.

Most interactions were ranked as non-critical by PVCA (87\%). Because of the grading system of the PVCA, this means that $87 \%$ of the interactions have the same rank: 1 . Therefore, information about each interaction and its criticality in comparison to other non-critical interactions could get lost. 
Here, intermediate ranks especially in the non-critical rank may help to further understand the individual criticality of each encounter.

\subsection{Limitations}

Even though data collection was done for over a week, the observation period was relatively small in comparison to the generally low frequency of crashes. Thus, no crashes and barely any critical interactions were found, resulting in a relatively low variance of PVCA and STCT rankings. This may have led to biases in the statistical analysis of the correlation between the methods. This effect might have been especially pronounced, as all non-critical interactions are labelled as rank 1 in the PVCA. Furthermore, because of the small amount of critical ranked interactions the analysis of different ranked interactions could only be done descriptive. Therefore, in future research the effect of the differences of the methods in other shared spaces with more critical interactions should be scrutinised.

For trajectory data collection, the AIM Mobile Unit was used. This system is able to observe a relatively large road environment, can detect and classify road users, and derive their trajectories. Unlike other naturalistic observation methods, such as field operational tests (FOT) or naturalistic driving studies (NDS), data collection is not done for individual road users only, but an observation space is filmed to investigate interactions of all road users at this particular section of the road environment. But while extensive advancements have been made in the computer vision enhanced analysis of road user behaviour, a number of shortcomings remain (Artan, Bulan, Loce, \& Paul, 2014; Lin, Deng, Albers, \& Siebert, 2020; Siebert \& Lin, 2020). In this study, a relatively high number of encounters had to be discarded, due to interruptions of the detection or difficulties in the discrimination between different road users. Especially, the difficulties in discrimination between different road users might have led to biases in the selection of interactions, as this problem occurred frequently when they were in close proximity to each other. Thus, situations, which could have been ranked as critical, might have been excluded.

The human annotation of the data and the evaluation of interrater reliability scores revealed a mixed result. While the agreement scores for the annotation of pedestrians' gender was high, with an agreement of over $90 \%$, agreement was lower for pedestrians' age categories. Raters agreed upon only one third of the observations. Human annotated changes in direction proved to be most problematic, with one observer identifying four changes in directions which the other did not find. For future research, clear, easy to follow definitions and instructions for the identification and registration of changes in directions are necessary.

\section{CONCLUSION}

In this study, the high agreement between criticality ratings provided by the STCT and the PVCA found by Kaparias et al. (2010) cannot be confirmed. Results indicate that differences in the focus and number of factors have an influence on the criticality rankings. Especially, situations with a high TTI and a small DTI were ranked differently. However, in a shared space, where mainly non-motorised road users can move freely in any direction, spatial distance (DTI) should be included in addition to TTI. Due to the small number of critical encounters, the investigation of the differences in the differently ranked encounters could only be analysed descriptively. Therefore, future studies should aim to collect larger sample sizes in more diverse shared space environments to be able to statistically confirm our here found results. Considering all mentioned advantages and disadvantages of both traffic conflict techniques, we conclude that the existing techniques are not fully suitable for analysing the severity of pedestrianvehicle encounters. Further research is needed to identify other factors influencing the degree of severity of pedestrianvehicle encounters.

\section{Acknowledgements}

We would like to thank Katharina Schuler for supporting the video annotation and Prof. Dr. Dietrich Manzey for his supervisory academic support.

\section{REFERENCES}

Artan, Y., Bulan, O., Loce, R. P., \& Paul, P. (2014). Driver Cell Phone Usage Detection From HOV/HOT NIR Images. Proceedings of the IEEE Conference on Computer Vision and Pattern Recognition Workshops, 225-230.

Barbosa, H. M., Tight, M. R., \& May, A. D. (2000). A model of speed profiles for traffic calmed roads. Transportation Research Part a: Policy and Practice, 34(2), 103-123. https://doi.org/10.1016/ S0965-8564(98)00067-6

Bortz, J., \& Schuster, C. (2010). Statistik für Human- und Sozialwissenschaftler. Berlin, Heidelberg: Springer Berlin Heidelberg. https://doi.org/10.1007/978-3-642-12770-0

Engwicht, D. (2005). Mental Speed Bumps: The smarter way to tame traffic. Sydney, Australien: Envirobook.

Follmer, R., \& Gruschwitz, D. (2019). Mobilität in Deutschland: MiD Kurzreport. Ausgabe 4.0 Studie von infas, DLR, IVT und infas 360 im Auftrag des Bundesministers für Verkehr und digitale Infrastrukt. Bonn, Berlin. Retrieved from https://www.bmvi. de/SharedDocs/DE/Anlage/G/mid-2017-kurzreport.pdf? blob=publicationFile

Gerlach, J., Boenke, D., Leven, J., \& Methorst, R. (2008). Sinn und Unsinn von Shared Space-Zur Versachlichung einer populären Gestaltungsphilosophie; Teil 1. Retrieved from http://www.th-owl.de/fb3/fileadmin/stephan_rainer/Shared Space/SVT 61-65.pdf

Hamilton-Baillie, B. (2008). Shared space: Reconciling people, places and traffic. Built Environment, 34(2), 161-181.

Hamilton-Baillie, B., \& Jones, P. (2005). Improving traffic behaviour and safety through urban design. Proceedings of the Institution of Civil Engineers - Civil Engineering, 158(5), 39-47. https://doi.org/10.1680/cien.2005.158.5.39

Hydén, C. (1987). The development of a method for traffic safety evaluation: The Swedish Traffic-Conflicts Technique (Dissertation). Lund University, Sweden. https://doi.org/10.1007/978-3-642-82109-7 12

Jacobsen, P. L. (2015). Safety in numbers: more walkers and bicyclists, safer walking and bicycling. Injury Prevention, 21(4), 271-275.

Jakobowsky, C. (2020). Neue Wege im Straßenverkehr? Untersuchung von Interaktionen zwischen Fußgänger * innen und Autofahrer* innen im Shared space (Master Thesis). Technische Universität Berlin, Berlin.

Jakobowsky, C., Siebert, F., Schießl, C., Junghans, M., \& Dotzauer, M. (2021). Why so serious? - Comparing two traffic conflict techniques for assessing encounters in shared space. https://doi.org/10.17605/OSF.IO/CZTVN

Kaparias, I., Bell, M. G. H., Biagioli, T., Bellezza, L., \& Mount, B. (2015). Behavioural analysis of interactions between pedestrians and vehicles in street designs with elements of shared space. Transportation Research Part F: Traffic Psychology and Behaviour, 30, 115-127. https://doi.org/10.1016/j. trf.2015.02.009

Kaparias, I., Bell, M., Dong, W., Sastrawinata, A., Singh, A., Wang, X., \& Mount, B. (2013). Analysis of Pedestrian-Vehicle Traffic Conflicts in Street Designs with Elements of Shared Space. Transportation Research Record: Journal of the Transportation Research Board, 2393(1), 21-30. https://doi.org/10.3141/2393-03 
Kaparias, I., Bell, M. G. H., Greensted, J., Cheng, S., Miri, A., Taylor, C., \& Mount, B. (2010). Development and Implementation of a Vehicle-Pedestrian Conflict Analysis Method: Adaptation of a Vehicle-Vehicle Technique.

Transportation Research Record, 2198(1), 75-82. https://doi.org/10.3141/2198-09

Karndacharuk, A., Wilson, D. J., \& Dunn, R. C. M. (2013). Analysis of Pedestrian Performance in Shared-Space Environments. Transportation Research Record, 2393(1), 1-11. https://doi.org/10.3141/2393-01

Karndacharuk, A., Wilson, D. J., \& Dunn, R. C. M. (2014a). A review of the evolution of shared (street) space concepts in urban environments. Transport Reviews, 34(2), 190-220.

Karndacharuk, A., Wilson, D. J., \& Dunn, R. C. M. (2014b). Safety performance study of shared pedestrian and vehicle space in New Zealand. Transportation Research Record, 2464(1), 1-10.

Laureshyn, A., \& Varhelyi, A. (2018). The Swedish Traffic Conflict Technique: observer's manual. Retrieved from https://www.indev-project.eu/InDeV/EN/Documents/pdf/TCTOM.pdf? blob=publicationFile\&v=2

Lin, H., Deng, J. D., Albers, D., \& Siebert, F. W. (2020). Helmet Use Detection of Tracked Motorcycles Using CNN-Based Multi-Task Learning. IEEE Access, 8, 162073-162084.

Monderman, H., Clarke, E., \& Baillie, B. H. (2006). Shared Space: The alternative approach to calming traffic. Traffic Engineering and Control, 47(8), 290-292.

Moody, S., \& Melia, S. (2011). Shared space: Implications of recent research for transport policy.
Schönauer, R., Stubenschrott, M., Schrom-Feiertag, H., \& Menšik, K. (2012). Social and spatial behaviour in Shared Spaces. Proceedings REAL CORP 2012, 759-767.

Shbeeb, L. (2000). Development of traffic conflicts technique for different environments: A comparative study of pedestrian conflicts in Sweden and Jordan (Doctoral thesis). Lund University.

Siebert, F. W., \& Lin, H. (2020). Detecting motorcycle helmet use with deep learning. Accident Analysis \& Prevention, 134, 105319.

Statistisches Bundesamt (2018). Unfallentwicklung auf deutschen Straßen 2017: Begleitmaterial zur Pressekonferenz am 12.Juli 2018 in Berlin. Wiesbaden. Retrieved from https://www.destatis.de/DE/Presse/Pressekonferenzen/2018/ Verkehrsunfaelle-2017/pressebroschuere-unfallentwicklung. pdf? blob=publicationFile

Statistisches Bundesamt (2020). 6,6 \% weniger Verkehrstote im Jahr 2019. Retrieved from https://www.destatis.de/DE/Presse/ Pressemitteilungen/2020/02/PD20 061 46241.html

Swain, J. (1987). Highway safety: The traffic conflict technique. London.

Tacchetti, M. (2018, December 5). User Guide for ELAN Linguistik Annotator. Retrieved from https://www.mpi.nl/corpus/ manuals/manual-elan ug.pdf

World Health Organization. (2009). Global status report on road safety: time for action: World Health Organization. Retrieved from https://apps.who.int/iris/bitstream/ handle/10665/44122/9789241563840 eng.pdf?sequence $=1$

\section{APPENDIX A. PVCA GRADING SYSTEM}

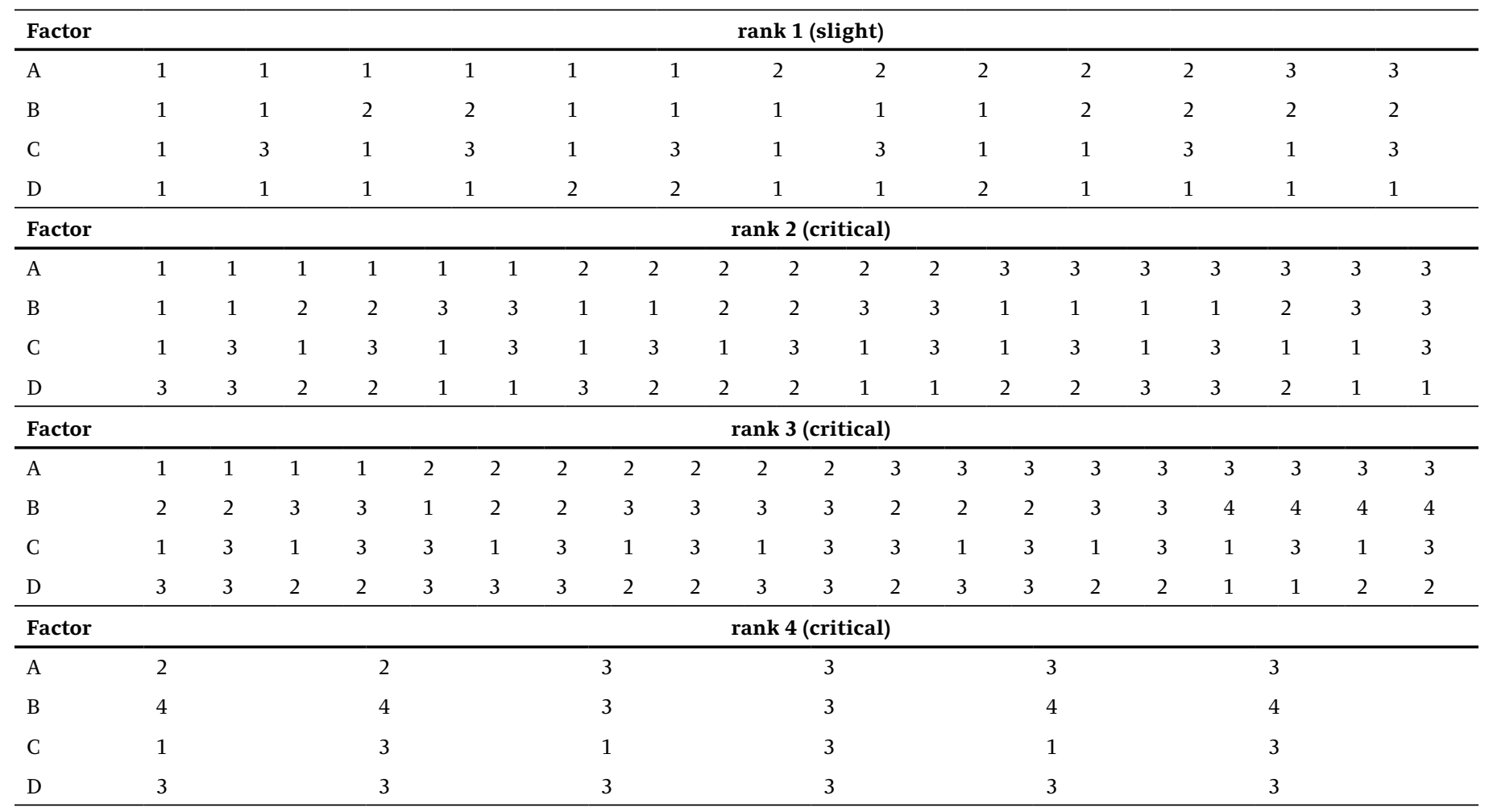

Table 3. Grading system of the PVCA (after Kaparias et al., 2013) 